\title{
REGULARIZATION OF NEARLY SINGULAR INTEGRALS IN THE BOUNDARY ELEMENT ANALYSIS FOR INTERIOR ANISOTROPIC THERMAL FIELD NEAR THE BOUNDARY
}

\author{
Yui-Chuin Shiah* and Yi-Shiau Shih
}

\begin{abstract}
In this paper the previously developed scheme of integration by parts to treat the boundary thermal field is applied to calculate the interior thermal field. Also, the scheme is extended further to regularize the strongly singular integral appearing in the boundary integral equation for interior calculations of the heat fluxes. Moreover, the present work develops a semi-analytical integration scheme to regularize the hypersingular integral for the interior heat-flux calculations. All formulations are derived for elements of arbitrary orders with general interpolation. At the end, the veracity of the scheme is illustrated by numerical examples.
\end{abstract}

Key Words: anisotropic thermal field, nearly singular integrals, interior thermal fields.

\section{INTRODUCTION}

As a result of extensive applications of anisotropic materials in engineering industries, the problem of anisotropic heat conduction has drawn substantial research since the early 1960 's. Although some analytical works in this regard have been reported over the years (see e.g. Tauchert and Akoz, 1975, Mulholland and Gupta, 1977, Chang, 1977), recourse to numerical tools, such as the finite element method (FEM) and the boundary element method (BEM), is still necessary for practical engineering problems. However, pertinent numerical works for the problem still remain relatively scarce as compared with those for isotropic media.

Recently, owing to its distinguishing feature, that only the boundary needs to be discretised, the BEM, used in the current study, has received much attention from researchers. However, difficulty will arise if the BEM is used to calculate the thermal field of interior points near the boundary. The source of this difficulty lies in the nearly singular integrals of the boundary integral equation (BIE) for calculating the interior thermal field. As already known, for computing temperature,

*Corresponding author. (Tel: 886-4-24517250 ext. 3956; Email: ycshiah@fcu.edu.tw)

The authors are with the Department of Aerospace and Systems Engineering, Feng Chia University, Taichung 407, Taiwan. the weakly singular integral of singular order $\ln (r)$ and the strongly singular integral with order $1 / r$, where $r$ is the distance between the source point and the integration point, will be involved. Even more challenging for interior calculations, computation of heat fluxes shall involve evaluations of the strongly singular integral and the hyper-singular integral with order (1/ $r^{2}$ ). When the interior points of interest are close to the boundary such that the order of the distance $r$ is much smaller than that of the element under integration, difficulty will arise in numerically integrating these integrals. Over the years, various BEM schemes have been proposed to overcome the nearly singular problem. Since there are too many pertinent works to be mentioned herein as a thorough literature review, only a few of them are reviewed as examples. Cruse and Aithal (1993) proposed a semi-analytical approach using Taylor series expansions for the kernels. Huang and Cruse (1993) presented another approach taking a coordinate transformation to relax nearly singular kernels. Other approaches include Gaussian integration with fine subdivisions, kernel cancellation methods (Nakagawa, 1993), the auxiliary surface of "tent" method (Lutz et $a l ., 1992)$, and the line integral method (Krishnasamy et al., 1994, Liu et al., 1993, and Liu, 1998). Sladek et al. (2001) proposed a semi-analytical integration scheme to deal with the logarithmic singularity in BEM. By applying the scheme of integration by parts, 
Zou et al. (2003) efficiently treated the nearly singular integrals for isotropic potential problems. More regularization algorithms can be referred to the references by Chen et al. (2001) and Kisu and Kawahara (1988). For more complete reviews on the role of hypersingularity, the reader may refer to , for example, Tanaka, et al. (1994), Chen and Hong (1999). A dual integral formulation that has regularized the singularities for the Laplace potential problem with a corner was derived by Chen and Hong (1994) using the contour approach surrounding the singularity. Very recently, the problem of the boundary layer effect has also been investigated by Chen et al. (2006a, 2006b), who have derived null-field integral equations for a medium containing circular cavities. By introducing the concept of degenerate kernels, Chen et al. (2006a) transformed the singular integrals into series sum when the null-field point was moved to the boundary.

In this article, the scheme of integration by parts is applied to treat the weakly singular integral, for which a general high-order interpolation of any families, such as the Serendipity, Lagrange, and Hermite, is considered. Additionally, for dealing with strongly singular and hyper-singular integrals, a semianalytical integration scheme is proposed. Successful implementation of the derived formulations is verified by numerical examples, where comparison with ANSYS analysis is made.

\section{BIE OF THE ANISOTROPIC THERMAL FIELD AT INTERIOR POINTS}

As already well established in the literature, associated 2D heat conduction in anisotropic media is governed by the so called Euler's equation,

$$
K_{i j} T_{i j}=0,(i, j=1,2),
$$

where $T$ stands for the temperature change; $K_{i j}$ are the anisotropic coefficients defined in the $x_{1}-x_{2}$ coordinate system. The analysis can be significantly simplified in the case of orthotropy, where the crossderivative terms are absent. As a result, a commonly adopted approach to numerically treat the fully anisotropic problem is to, first, determine the principal axes $\left(\zeta_{1}, \zeta_{2}\right)$ by rotating the original Cartesian axes such that the cross-derivative terms will disappear. Another attractive approach to treat the anisotropic problem is to employ a coordinate transformation such that $T$ in the transformed domain is governed by the standard Laplace's equation. In their efforts to formulate an exact transformation of the volume integral associated with thermal effects into surface integrals for plane anisotropic thermoelasticity in BEM, Shiah and Tan (1998) also developed a similar transformation and applied it to numerically treat the two-dimensional anisotropic problem in the BEM field theory. The main advantage of such a BEM treatment lies in the fact that the anisotropic field problem can be easily solved using any readily available BEM codes for 'isotropic' potential theory with relatively minor program modifications. The linear transformation/inverse transformation takes the following form,

$$
\begin{aligned}
& {\left[\begin{array}{ll}
\hat{x}_{1} & \hat{x}_{2}
\end{array}\right]^{T}=\left[F\left(K_{i j}\right)\right]\left[\begin{array}{ll}
x_{1} & x_{2}
\end{array}\right]^{T},} \\
& {\left[\begin{array}{ll}
x_{1} & x_{2}
\end{array}\right]^{T}=\left[F^{-1}\left(K_{i j}\right)\right]\left[\begin{array}{ll}
\hat{x}_{1} & \hat{x}_{2}
\end{array}\right]^{T}}
\end{aligned}
$$

where $\left[F\left(K_{i j}\right)\right]$ is the transformation (and $\left[F^{-1}\left(K_{i j}\right)\right]$ the inverse transformation) matrix, given by

$$
\begin{aligned}
& \boldsymbol{F}=\left(\begin{array}{cc}
\sqrt{\Delta} / K_{11} & 0 \\
-K_{12} / K_{11} & 1
\end{array}\right), \boldsymbol{F}^{-1}=\left(\begin{array}{cc}
K_{11} / \sqrt{\Delta} & 0 \\
K_{12} / \sqrt{\Delta} & 1
\end{array}\right), \\
& \Delta=K_{11} K_{22}-K_{12}^{2}
\end{aligned}
$$

With this transformation, the anisotropic field is now governed by the standard Laplace equation. For equivalently isotropic field problems, the temperature change $T$ and its normal gradient $q$ at interior points are related by

$$
\begin{aligned}
T(P)= & \int_{S} q(Q) T^{*}(P, Q) d \hat{S}(Q) \\
& -\int_{S} T(Q) Q^{*}(P, Q) d \hat{S}(Q),
\end{aligned}
$$

where $P$ and $Q$ are the source and field points on the boundary, respectively; $T^{*}(P, Q)$ and $Q^{*}(P, Q)$ represent the fundamental solutions for the temperature and its normal gradient, given by

$$
T^{*}(P, Q)=\frac{1}{2 \pi} \log \frac{1}{r}, Q^{*}(P, Q)=\frac{-1}{2 \pi r} \frac{d r}{d n}
$$

where $r$ is the distance between the source point $P$ and the field point $Q$ on the element under integration. To numerically solve the BIE, the boundary surface is discretised into a number of segments or elements, say $M$ elements, with a total of $N$ nodes. Following the usual interpolation process for $n$-order elements, one may have nodal values of coordinates, temperature, and temperature gradients expressed in terms of the local coordinate $\xi \in[-1,1]$ as

$$
\begin{aligned}
& x_{j}(\xi)=\sum_{c=1}^{n} N^{(c)}(\xi) x_{j}^{(c)}, T(\xi)=\sum_{c=1}^{n} N^{(c)}(\xi) T^{(c)}, \\
& q(\xi)=\sum_{c=1}^{n} N^{(c)}(\xi) q^{(c)}
\end{aligned}
$$


where $N^{(c)}$, the shape functions of $n$ degrees, has a general form

$$
N^{(c)}(\xi)=\sum_{m=0}^{n} \alpha_{m}^{(c)} \xi^{m}
$$

Equation (8) is for general interpolations of any kind of interpolation families. Substitution of Eqs. (6), (7), and (8) into Eq. (5) results in a discretised BIE form,

$$
\begin{aligned}
T\left(P^{a}\right)= & \sum_{b=1}^{M} \sum_{c=1}^{n+1}{ }^{b} q^{c} \int_{-1}^{1} \frac{-1}{2 \pi} \log ^{b} r N^{c}(\xi) \hat{J}(\xi) d \xi \\
& +\sum_{b=1}^{M} \sum_{c=1}^{n+1}{ }^{b} T^{c} \int_{-1}^{1} \frac{1}{2 \pi^{b} r} \frac{d^{b} r}{d n} N^{c}(\xi) \hat{J}(\xi) d \xi
\end{aligned}
$$

where the superscript $a$ represents the $a$-th global node of the boundary mesh, $b$ denotes the $b$-th element, and $c$ is the $c$-th node of the element. In Eq. $(8), \hat{J}(\xi)$ is the Jacobian, given by

$$
\hat{J}(\xi)=\sqrt{\left(\sum_{i=1}^{n+1} \sum_{m=1}^{n} m \alpha_{m}^{(i)} \xi^{m-1} \hat{x}_{1}^{(i)}\right)^{2}+\left(\sum_{i=1}^{n+1} \sum_{m=1}^{n} m \alpha_{m}^{(i)} \xi^{m-1} \hat{x}_{2}^{(i)}\right)^{2}}
$$

Apparently, the first and the second integral terms in Eq. (8) are weakly singular and strongly singular, respectively. As derived in the authors' previous work (Shiah and Shih, 2005), the thermal BIE can be regularized using the scheme of integration by parts and a semi-analytical approach. The regularized BIE for the interior temperature field can be written as

$$
\begin{aligned}
T\left(P^{a}\right)= & \sum_{b=1}^{M} \sum_{c=1}^{n+1} \frac{-b}{4 \pi}\left[W(\xi) L(\xi)-\int_{-1}^{1} L(\xi) \Omega^{(c)}(\xi) d \xi\right] \\
& +\sum_{b=1}^{M} \sum_{c=1}^{n+1} \frac{b}{2 \pi} T^{c} \sum_{l=1}^{2 n} \sum_{m=0}^{n} H_{l} \alpha_{m}^{(c)}\left[\sum_{k=0}^{m-1} \frac{V_{l}^{k} \xi^{m-k}}{(m-k)}\right. \\
& \left.+V_{l}^{m} \log \left(\xi-V_{l}\right)\right]\left.\right|_{-1} ^{1}
\end{aligned}
$$

where $W(\xi), L(\xi), \Omega^{(c)}(\xi)$ are given by

$$
\begin{aligned}
& W(\xi)=\sum_{t=0}^{n} \alpha_{t}^{(c)} \xi^{t} \hat{J}(\xi) \\
& L(\xi)=\xi \log \left(F_{2 n}\right)+\sum_{l=1}^{2 n}\left(\xi-V_{l}\right)\left[\log \left(\xi-V_{l}\right)-1\right]
\end{aligned}
$$

$$
\Omega^{(c)}(\xi)=\sum_{t=1}^{n} t \alpha_{t}^{(c)} \xi^{t-1} \hat{J}(\xi)+\sum_{t=0}^{n} t \alpha_{t}^{(c)} \xi^{t} \widehat{J}^{\prime}(\xi)
$$

$$
H_{l}=\frac{\sum_{i, j=1}^{n+1} \sum_{m=0}^{n} \sum_{t=1}^{n} t \alpha_{t}^{(j)}\left(\beta_{1 m}^{(i)} \hat{x}_{1}^{(i)} \hat{x}_{2}^{(j)}-\beta_{2 m}^{(i)} \hat{x}_{2}^{(i)} \hat{x}_{1}^{(j)}\right) V_{l}^{m+t-1-2 n}}{F_{2 n} \prod_{k=1}^{2 n}\left(1-V_{k} / V_{l}+V_{k} \delta_{k l} / V_{l}\right)}
$$

In Eq. (10), $V_{l}$ are $2 n$ roots of the following polynomial,

$$
\sum_{k=0}^{2 n} \frac{F_{2 n-k}}{F_{2 n}} \xi^{2 n-k}=0
$$

where the coefficients $F_{2 n-k}$ are defined by

$$
\begin{aligned}
& F_{2 n-k}=\sum_{m=0}^{k} \sum_{i, j=1}^{n+1} \beta_{l(n-m)}^{(i)} \beta_{l(n+m-k)}^{(j)} \hat{x}_{l}^{(i)} \hat{x}_{l}^{(j)}, \\
& \beta_{j m}^{(i)}= \begin{cases}\alpha_{m}^{(i)} \hat{x}_{j}^{(i)}, & \text { for } m \neq 0 \\
\alpha_{0}^{(i)} \hat{x}_{j}^{(i)}-\hat{x}_{p j}, & \text { for } m=0\end{cases}
\end{aligned}
$$

and $\hat{x}_{j}^{(i)}$ refers to the mapped coordinates at the $i$-th node of the element. In Eq. (11c), the explicit form $\hat{J}^{\prime}$ $(\xi)$ is written as

$$
\hat{J}^{\prime}(\xi)=\frac{\sum_{i, j=1}^{n+1} \sum_{m=1}^{n} \sum_{k=2}^{n} m k(k-1) \alpha_{m}^{(i)} \alpha_{k}^{(j)} \hat{x}_{l}^{(i)} \hat{x}_{l}^{(j)} \xi^{m+k-3}}{\hat{J}(\xi)} .
$$

\section{REGULARIZATION OF THE BIE FOR CALCULATING INTERNAL HEAT FLUXES}

As one of the goals for interior calculations, the internal heat fluxes also need to be determined. By definition, the heat fluxes in anisotropic media, $Q_{i}$, are given by

$$
Q_{i}=K_{i j} T_{, j}
$$


which, in the mapped plane, can be explicitly written as

$$
Q_{i}=K_{i 1}\left(\frac{\partial T}{\partial \hat{x}_{1}} \frac{\sqrt{\Delta}}{K_{11}}-\frac{\partial T}{\partial \hat{x}_{2}} \frac{K_{12}}{K_{11}}\right)+K_{i 2} \frac{\partial T}{\partial \hat{x}_{2}} .
$$

Apparently, determination of the heat fluxes via the above equations needs to resort to the computation of $\partial T / \partial \hat{x}_{1}, \partial T / \partial \hat{x}_{2}$ by the following differentiated BIE form,

$$
\begin{aligned}
\frac{d T\left(P^{a}\right)}{d \hat{x}_{p 1}}= & \sum_{b=1}^{M} \sum_{c=1}^{n+1} \frac{q^{c}}{2 \pi} \int_{-1}^{1} \frac{\left(\hat{x}_{1}-\hat{x}_{p 1}\right)}{\left(\hat{x}_{1}-\hat{x}_{p 1}\right)^{2}+\left(\hat{x}_{2}-\hat{x}_{p 2}\right)^{2}} N^{(c)}(\xi) \hat{J}(\xi) d \xi \\
& -\sum_{b=1}^{M} \sum_{c=1}^{n+1} \frac{{ }^{b} T^{c}}{2 \pi} \int_{-1}^{1} \frac{\left[\left(\hat{x}_{1}-\hat{x}_{p 1}\right)^{2}-\left(\hat{x}_{2}-\hat{x}_{p 2}\right)^{2}\right] \frac{d \hat{x}_{2}}{d \xi}-2\left(\hat{x}_{1}-\hat{x}_{p 1}\right)\left(\hat{x}_{2}-\hat{x}_{p 2}\right) \frac{d \hat{x}_{1}}{d \xi}}{\left[\left(\hat{x}_{1}-\hat{x}_{p 1}\right)^{2}+\left(\hat{x}_{2}-\hat{x}_{p 2}\right)^{2}\right]^{2}} N^{(c)}(\xi) d \xi \\
\frac{d T\left(P^{a}\right)}{d \hat{x}_{p 2}}= & \sum_{b=1}^{M} \sum_{c=1}^{n+1} \frac{q^{c}}{2 \pi} \int_{-1}^{1} \frac{\left(\hat{x}_{2}-\hat{x}_{p 2}\right)}{\left(\hat{x}_{1}-\hat{x}_{p 1}\right)^{2}+\left(\hat{x}_{2}-\hat{x}_{p 2}\right)^{2}} N^{(c)}(\xi) \hat{J}(\xi) d \xi \\
& -\sum_{b=1}^{M} \sum_{c=1}^{n+1} \frac{b}{2 \pi} \int_{-1}^{1} \frac{\left.2\left(\hat{x}_{1}-\hat{x}_{p 1}\right)\left(\hat{x}_{2}-\hat{x}_{p 2}\right) \frac{d \hat{x}_{2}}{d \xi}+\left[\left(\hat{x}_{1}-\hat{x}_{p 1}\right)^{2}+\left(\hat{x}_{2}-\hat{x}_{p 2}\right)^{2}\right]^{2}-\hat{x}_{p 2}\right)^{2} \frac{d \hat{x}_{1}}{d \xi}}{2 \pi} N^{(c)}(\xi) d \xi .
\end{aligned}
$$

It is obvious that the first integral in the above equations is strongly singular, while the second one is hyper-singular. In the same manner as before for the thermal BIE, the integrals are treated separately by different schemes. By use of the interpolation given in Eqs. (7) and (8), the strongly singular integral in Eq. (17a) can be rewritten as

$$
\begin{aligned}
& \int_{-1}^{1} \frac{\left(\hat{x}_{1}-\hat{x}_{p 1}\right)}{\left(\hat{x}_{1}-\hat{x}_{p 1}\right)^{2}+\left(\hat{x}_{2}-\hat{x}_{p 2}\right)^{2}} N^{(c)}(\xi) \hat{J}(\xi) d \xi \\
& =\int_{-1}^{1} \frac{\sum_{i=1}^{n+1} \sum_{m, j=0}^{n} \beta_{1 m}^{(i)} \hat{x}_{1}^{(i)} \alpha_{j}^{(c)} \xi^{m+j}}{F_{2 n} \prod_{l=1}^{2 n}\left(\xi-V_{l}\right)} \hat{J}(\xi) d \xi \\
& =\int_{-1}^{1}\left(\begin{array}{c}
\sum_{i=1}^{n+1} \frac{\beta_{1 n}^{(i)} \hat{x}_{1}^{(i)} \alpha_{n}^{(c)}}{F_{2 n}} \\
+\frac{\sum_{i=1}^{n+1} \hat{x}_{1}^{(i)}\left[-\sum_{m=0}^{2 n-1} \beta_{1 n}^{(i)} \alpha_{n}^{(c)} F_{m} \xi^{m} / F_{2 n}\right.}{F_{2 n} \prod_{l=1}^{2 n}\left(\xi-V_{l}\right)}
\end{array}\right)
\end{aligned}
$$

The first integral term in the last equation of Eq. (18) has no singularity since its Jacobian shall not approach zero under the nearly singular condition. Therefore, it can be directly integrated using any 
numerical integration scheme such as Gauss integration. However, the problem of nearly singular integral shall arise for the last integral term in Eq.
(18). For simplifying the regularization process, its integrand can be rearranged to have the form,

$$
\frac{\sum_{i=1}^{n+1} \hat{x}_{1}^{(i)}\left[\begin{array}{c}
\left(\beta_{1 n}^{(i)} \alpha_{n-1}^{(c)}+\beta_{1 n-1}^{(i)} \alpha_{n}^{(c)}\right) \xi^{2 n-1}+\sum_{m, j=0}^{n-1} \beta_{1 m}^{(i)} \alpha_{j}^{(c)} \xi^{m+j} \\
-\sum_{m=0}^{2 n-1} \beta_{1 n}^{(i)} \alpha_{n}^{(c)} F_{m} \xi^{m} / F_{2 n}
\end{array}\right]}{F_{2 n} \prod_{l=1}^{2 n}\left(\xi-V_{l}\right)} \hat{J}(\xi)=\left(\sum_{l=1}^{2 n} \frac{G_{l}}{\left(\xi-V_{l}\right)}\right) \hat{J}(\xi)
$$

where the constant $G_{l}$ is given by

$$
G_{l}=\frac{\sum_{i=1}^{n+1} \hat{x}_{1}^{(i)}\left[\begin{array}{c}
\left(\beta_{1 n}^{(i)} \alpha_{n-1}^{(c)}+\beta_{1 n-1}^{(i)} \alpha_{n}^{(c)}\right) V_{l}^{-1}+\sum_{m, j=0}^{n-1} \beta_{1 m}^{(i)} \alpha_{j}^{(c)} V_{l}^{m+j-2 n} \\
-\sum_{m=0}^{2 n-1} \beta_{1 n}^{(i)} \alpha_{n}^{(c)} F_{m} V_{l}^{m-2 n} / F_{2 n}
\end{array}\right]}{F_{2 n} \prod_{k=1}^{2 n}\left(1-V_{k} / V_{l}+V_{k} \delta_{k l} / V_{l}\right)} .
$$

Applying the scheme of integration by parts, one may obtain

$$
\begin{aligned}
& \int_{-1}^{1}\left(\sum_{l=1}^{2 n} \frac{G_{l}}{\left(\xi-V_{l}\right)}\right) \hat{J}(\xi) d \xi \\
& =\sum_{l=1}^{2 n} G_{l}\left(\left.\hat{J}(\xi) \log \left(\xi-V_{l}\right)\right|_{-1} ^{1}-\int_{-1}^{1} \hat{J}^{\prime}(\xi) \log \left(\xi-V_{l}\right) d \xi\right),
\end{aligned}
$$

where the singularity order has been reduced to be weakly singular. To fully remove the singularity, the weakly singular integral in Eq. (21) has to be integrated by parts again to give

$$
\begin{aligned}
& \int_{-1}^{1}\left(\sum_{l=1}^{2 n} \frac{G_{l}}{\left(\xi-V_{l}\right)}\right) \hat{J}(\xi) d \xi \\
& =\sum_{l=1}^{2 n} G_{l}\left(\left\{\left[\hat{J}(\xi)-\hat{J}^{\prime}(\xi)\left(\xi-V_{l}\right)\right] \log \left(\xi-V_{l}\right)\right.\right. \\
& \left.\quad+\hat{J}^{\prime}(\xi)\left(\xi-V_{l}\right)\right\}\left.\right|_{-1} ^{1}+\int_{-1}^{1} \hat{J}^{\prime \prime}(\xi)\left(\xi-V_{l}\right) \\
& \left.\quad \cdot\left[\log \left(\xi-V_{l}\right)-1\right] d \xi\right)
\end{aligned}
$$

where $\widehat{J}^{\prime \prime}(\xi)$ represents the differentiated Jacobian of the second order, explicitly given by

$$
\begin{array}{r}
\hat{J}^{\prime \prime}(\xi)=\frac{\sum_{i, j=1}^{n+1} \sum_{s=1}^{n} \sum_{t=2}^{n} s t(t-1)(s+t-3) \alpha_{s}^{(i)} \alpha_{t}^{(j)} \hat{x}_{l}^{(i)} \hat{x}_{l}^{(j)} \xi^{s+t-4}}{\hat{J}(\xi)} \\
-\frac{\sum_{i, j=1}^{n+1} \sum_{m=1}^{n} \sum_{k=2}^{n} m k(k-1) \alpha_{m}^{(i)} \alpha_{k}^{(j)} \hat{x}_{l}^{(i)} \hat{x}_{l}^{(j)} \xi^{m+k-3} \hat{J}^{\prime}(\xi)}{\hat{J}^{2}(\xi)},
\end{array}
$$

where $s, t$ must satisfy $s+t \geq 4$. With its singularity order reduced, Eq. (22) can now be evaluated using a regular numerical integration scheme to give accurate results. The strongly singular integral appearing in Eq. (17b) can be treated in the same way as described above. The derived formulae can be applied by simply replacing $\hat{x}_{1}^{(i)}$ by $\hat{x}_{2}^{(i)}$ and $\beta_{1 m}^{(i)}$ by $\beta_{2 m}^{(i)}$ in the numerator of Eq. (18) and in the subsequent derivations.

Next, the goal is to regularize the hyper-singular integrals in Eqs. (17a) and (17b). By interpolating all spatial coordinates using Eq. (8), the hyper-singular integral in Eq. (17a) can be written as 


$$
\int_{-1}^{1} \frac{\left[\left(\hat{x}_{1}-\hat{x}_{p 1}\right)^{2}-\left(\hat{x}_{2}-\hat{x}_{p 2}\right)^{2}\right] \frac{d \hat{x}_{2}}{d \xi}-2\left(\hat{x}_{1}-\hat{x}_{p 1}\right)\left(\hat{x}_{2}-\hat{x}_{p 2}\right) \frac{d \hat{x}_{1}}{d \xi}}{\left[\left(\hat{x}_{1}-\hat{x}_{p 1}\right)^{2}+\left(\hat{x}_{2}-\hat{x}_{p 2}\right)^{2}\right]^{2}} N^{(c)}(\xi) d \xi=\int_{-1}^{1} \frac{f_{1}(\xi)}{F_{2 n}^{2} \prod_{w=1}^{2 n}\left(\xi-V_{w}\right)^{2}} d \xi
$$

where $f_{1}(\xi)$ represents

$$
f_{1}(\xi)=\sum_{i, j, k=1}^{n+1} \sum_{l, m, s=0}^{n} \sum_{t=1}^{n} t \alpha_{t}^{(k)} \alpha_{s}^{(c)} \cdot\left(\begin{array}{r}
\beta_{1 l}^{(i)} \beta_{1 m}^{(j)} \hat{x}_{1}^{(i)} \hat{x}_{1}^{(j)} \hat{x}_{2}^{(k)} \\
-\beta_{2 l}^{(i)} \beta_{2 m}^{(j)} \hat{x}_{2}^{(i)} \hat{x}_{2}^{(j)} \hat{x}_{2}^{(k)} \\
-2 \beta_{1 l}^{(i)} \beta_{2 m}^{(j)} \hat{x}_{1}^{(i)} \hat{x}_{2}^{(j)} \hat{x}_{1}^{(k)}
\end{array}\right) \xi^{s+m+l+t-1}
$$

Obviously, the denominator is a polynomial function of $\xi$ with degrees $4 n$, and the polynomial in the numerator has degrees $4 n-1$. The integrand can be further processed to give

$$
\int_{-1}^{1} \frac{\left[\left(\hat{x}_{1}-\hat{x}_{p 1}\right)^{2}-\left(\hat{x}_{2}-\hat{x}_{p 2}\right)^{2}\right] \frac{d \hat{x}_{2}}{d \xi}-2\left(\hat{x}_{1}-\hat{x}_{p 1}\right)\left(\hat{x}_{2}-\hat{x}_{p 2}\right) \frac{d \hat{x}_{1}}{d \xi}}{\left[\left(\hat{x}_{1}-\hat{x}_{p 1}\right)^{2}+\left(\hat{x}_{2}-\hat{x}_{p 2}\right)^{2}\right]^{2}} N^{(c)}(\xi) d \xi=\int_{-1}^{1} \sum_{w=1}^{2 n}\left(\frac{C_{w 1}}{\left(\xi-V_{w}\right)}+\frac{C_{w 2}}{\left(\xi-V_{w}\right)^{2}}\right) d \xi
$$

where the constants $C_{w 1}, C_{w 2}$ are given by

$$
\begin{gathered}
C_{w 1}=\frac{f_{1}^{\prime}\left(V_{w}\right)-2 f_{1}\left(V_{w}\right) V_{w}^{-(4 n+1)} \sum_{k=1}^{2 n}\left(1-V_{k} / V_{w}+V_{k} \delta_{k w} / V_{w}\right)^{-1}}{F_{2 n}^{2} \prod_{k=1}^{2 n}\left(1-V_{k} / V_{w}+V_{k} \delta_{k w} / V_{w}\right)^{2}} \\
C_{w 2}=\frac{\sum_{i, j, k=1}^{n+1} \sum_{l, m, s=0}^{n} \sum_{t=1}^{n} t \alpha_{t}^{(k)} \alpha_{s}^{(c)}\left(\begin{array}{c}
\beta_{1 l}^{(i)} \beta_{1 m}^{(j)} \hat{x}_{1}^{(i)} \hat{x}_{1}^{(j)} \hat{x}_{2}^{(k)} \\
-\beta_{2 l}^{(i)} \beta_{2 m}^{(j)} \hat{x}_{2}^{(i)} \hat{x}_{2}^{(j)} \hat{x}_{2}^{(k)} \\
-2 \beta_{1 l}^{(i)} \beta_{2 m}^{(j)} \hat{x}_{1}^{(i)} \hat{x}_{2}^{(j)} \hat{x}_{1}^{(k)}
\end{array}\right) V_{w}^{s+m+l+t-4 n-1}}{F_{2 n}^{2} \prod_{k=1}^{2 n}\left(1-V_{k} / V_{w}+V_{k} \delta_{k l} / V_{w}\right)^{2}} .
\end{gathered}
$$

In Eq. (27a), the general form of $f_{1}^{\prime}(\xi)$, denoting differentiation of $f_{1}(\xi)$, is written as

$$
f_{1}^{\prime}(\xi)=\sum_{i, j, k=1}^{n+1} \sum_{l, m, s=0}^{n} \sum_{t=1}^{n} t(s+m+l+t-1) \alpha_{t}^{(k)} \alpha_{s}^{(c)} \cdot\left(\begin{array}{c}
\beta_{1 l}^{(i)} \beta_{1 m}^{(j)} \hat{x}_{1}^{(i)} \hat{x}_{1}^{(j)} \hat{x}_{2}^{(k)} \\
-\beta_{2 l}^{(i)} \beta_{2 m}^{(j)} \hat{x}_{2}^{(i)} \hat{x}_{2}^{(j)} \hat{x}_{2}^{(k)} \\
-2 \beta_{1 l}^{(i)} \beta_{2 m}^{(j)} \hat{x}_{1}^{(i)} \hat{x}_{2}^{(j)} \hat{x}_{1}^{(k)}
\end{array}\right) \xi^{s+m+l+t-2}
$$




$$
\begin{aligned}
& \int_{-1}^{1} \frac{\left[\left(\hat{x}_{1}-\hat{x}_{p 1}\right)^{2}-\left(\hat{x}_{2}-\hat{x}_{p 2}\right)^{2}\right] \frac{d \hat{x}_{2}}{d \xi}-2\left(\hat{x}_{1}-\hat{x}_{p 1}\right)\left(\hat{x}_{2}-\hat{x}_{p 2}\right) \frac{d \hat{x}_{1}}{d \xi}}{\left[\left(\hat{x}_{1}-\hat{x}_{p 1}\right)^{2}+\left(\hat{x}_{2}-\hat{x}_{p 2}\right)^{2}\right]^{2}} N^{(c)}(\xi) d \xi \\
& =\int_{-1}^{1} \sum_{w=1}^{2 n}\left(\frac{C_{w 1}}{\left(\xi-V_{w}\right)}+\frac{C_{w 2}}{\left(\xi-V_{w}\right)^{2}}\right) d \xi=\left.\sum_{w=1}^{2 n}\left[C_{w 1} \log \left(\xi-V_{w}\right)-\frac{C_{w 2}}{\left(\xi-V_{w}\right)}\right]\right|_{-1} ^{1}
\end{aligned}
$$

In a similar manner, the hyper-singular integral in Eq. (17b) can be treated likewise. Following the foregoing processes, one may rewrite the hyper-singular integral as

$$
\begin{aligned}
& \int_{-1}^{1} \frac{2\left(\hat{x}_{1}-\hat{x}_{p 1}\right)\left(\hat{x}_{2}-\hat{x}_{p 2}\right) \frac{d \hat{x}_{2}}{d \xi}+\left[\left(\hat{x}_{1}-\hat{x}_{p 1}\right)^{2}-\left(\hat{x}_{2}-\hat{x}_{p 2}\right)^{2}\right] \frac{d \hat{x}_{1}}{d \xi}}{\left[\left(\hat{x}_{1}-\hat{x}_{p 1}\right)^{2}+\left(\hat{x}_{2}-\hat{x}_{p 2}\right)^{2}\right]^{2}} N^{(c)}(\xi) d \xi \\
& =\int_{-1}^{1} \frac{f_{2}(\xi)}{F_{2 n}^{2} \prod_{w=1}^{2 n}\left(\xi-V_{w}\right)^{2}} d \xi=\int_{-1}^{1} \sum_{w=1}^{2 n}\left[\frac{D_{w 1}}{\left(\xi-V_{w}\right)}+\frac{D_{w 2}}{\left(\xi-V_{w}\right)^{2}}\right] d \xi
\end{aligned}
$$

where the function $f_{2}(\xi)$ is given by

$$
f_{2}(\xi)=\sum_{i, j, k=1}^{n+1} \sum_{l, m, s=0}^{n} \sum_{t=1}^{n} t \alpha_{t}^{(k)} \alpha_{s}^{(c)} \cdot\left(\begin{array}{c}
\beta_{1 l}^{(i)} \beta_{1 m}^{(j)} \hat{x}_{1}^{(i)} \hat{x}_{1}^{(j)} \hat{x}_{1}^{(k)} \\
-\beta_{2 l}^{(i)} \beta_{2 m}^{(j)} \hat{x}_{2}^{(i)} \hat{x}_{2}^{(j)} \hat{x}_{1}^{(k)} \\
2 \beta_{1 l}^{(i)} \beta_{2 m}^{(j)} \hat{x}_{1}^{(i)} \hat{x}_{2}^{(j)} \hat{x}_{2}^{(k)}
\end{array}\right) \xi^{s+m+l+t-1}
$$

and the constants $D_{w 1}, D_{w 2}$ are given by

$$
\begin{gathered}
D_{w 1}=\frac{f_{2}^{\prime}\left(V_{w}\right)-2 f_{2}\left(V_{w}\right) V_{w}^{-(4 n+1)} \sum_{k=1}^{2 n}\left(1-V_{k} / V_{w}+V_{k} \delta_{k w} / V_{w}\right)^{-1}}{F_{2 n}^{2} \prod_{k=1}^{2 n}\left(1-V_{k} / V_{w}+V_{k} \delta_{k w} / V_{w}\right)^{2}} \\
D_{w 2}=\frac{\sum_{i, j, k=1}^{n+1} \sum_{l, m, s=0}^{n} \sum_{t=1}^{n} t \alpha_{t}^{(k)} \alpha_{s}^{(c)}\left(\begin{array}{r}
\beta_{1 l}^{(i)} \beta_{1 m}^{(j)} \hat{x}_{1}^{(i)} \hat{x}_{1}^{(j)} \hat{x}_{1}^{(k)} \\
-\beta_{2 l}^{(i)} \beta_{2 m}^{(j)} \hat{x}_{2}^{(i)} \hat{x}_{2}^{(j)} \hat{x}_{1}^{(k)} \\
-2 \beta_{1 l}^{(i)} \beta_{2 m}^{(j)} \hat{x}_{1}^{(i)} \hat{x}_{2}^{(j)} \hat{x}_{2}^{(k)}
\end{array}\right) V_{w}^{s+m+l+t-4 n-1}}{F_{2 n}^{2} \prod_{k=1}^{2 n}\left(1-V_{k} / V_{w}+V_{k} \delta_{k l} / V_{w}\right)^{2}} .
\end{gathered}
$$

Sequentially, Eq. (30) can be analytically integrated to give (33) To this end, the singularity of all singular integrals has been fully removed. As a result, the regularized BIE of temperature gradients can be expressed as 


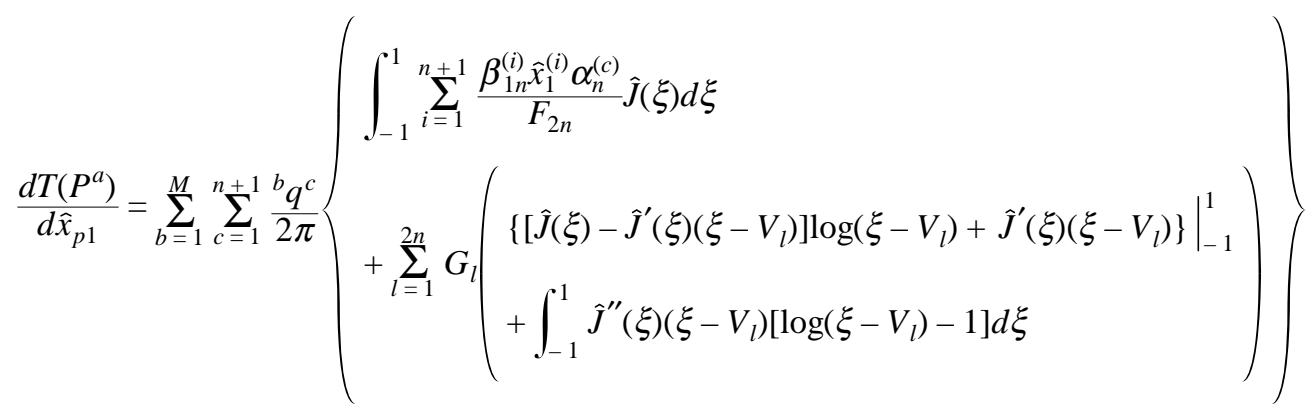

$$
\begin{aligned}
& -\left.\sum_{b=1}^{M} \sum_{c=1}^{n+1}{ }^{b} T^{c} \sum^{2} \sum_{w=1}^{2 n}\left[C_{w 1} \log \left(\xi-V_{w}\right)-\frac{C_{w 2}}{\left(\xi-V_{w}\right)}\right]\right|_{-1} ^{1}
\end{aligned}
$$

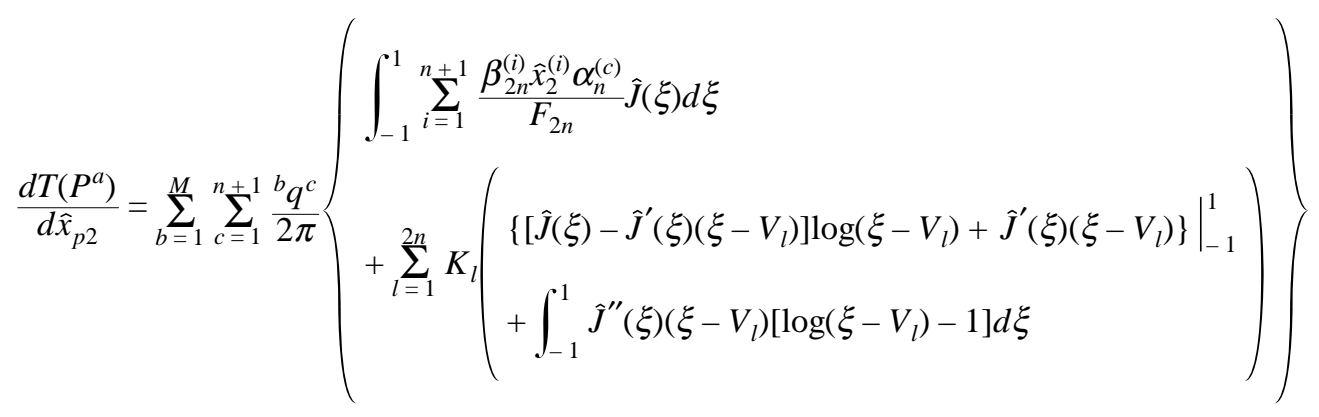

$$
\begin{aligned}
& -\left.\sum_{b=1}^{M} \sum_{c=1}^{n+1} \frac{{ }^{b}}{2} T^{c} \sum_{w=1}^{2 n}\left[D_{w 1} \log \left(\xi-V_{w}\right)-\frac{D_{w 2}}{\left(\xi-V_{w}\right)}\right]\right|_{-1} ^{1}
\end{aligned}
$$

In Eq. (34b), the constant $K_{l}$ is given by

$$
K_{l}=\frac{\sum_{i=1}^{n+1} \hat{x}_{2}^{(i)}\left[\begin{array}{l}
\left(\beta_{2 n}^{(i)} \alpha_{n-1}^{(c)}+\beta_{2 n-1}^{(i)} \alpha_{n}^{(c)}\right) V_{l}^{-1}+\sum_{m, j=0}^{n-1} \beta_{2 m}^{(i)} \alpha_{j}^{(c)} V_{l}^{m+j-2 n} \\
-\sum_{m=0}^{2 n-1} \beta_{2 n}^{(i)} \alpha_{n}^{(c)} F_{m} V_{l}^{m-2 n} / F_{2 n}
\end{array}\right]}{F_{2 n} \prod_{k=1}^{2 n}\left(1-V_{k} / V_{l}+V_{k} \delta_{k l} / V_{l}\right)} .
$$

Having calculated the temperature gradients in the mapped plane, one may readily obtain the heat fluxes defined in the physical plane using Eq. (16). In what follows, the veracity of the derived formulae will be demonstrated by two numerical examples.

\section{NUMERICAL EXAMPLES}

All formulae derived have been implemented in an existing computer program based on quadratic isoparametric elements $(n=2)$ using Lagrange interpolation. For demonstration of the proposed scheme, the first problem considered is a square glass/ epoxy plate with dimensions as shown in Fig. 1. The conductivity coefficients of the material with asterisk denoting the properties in the principal axes are

$$
K_{11}^{*} / K_{22}^{*}=3.46 / 0.35 \text {. }
$$

To account for the case with fully anisotropic properties, the principal axes are rotated with an angle $\theta=60^{\circ}$. For the boundary conditions, the top and 


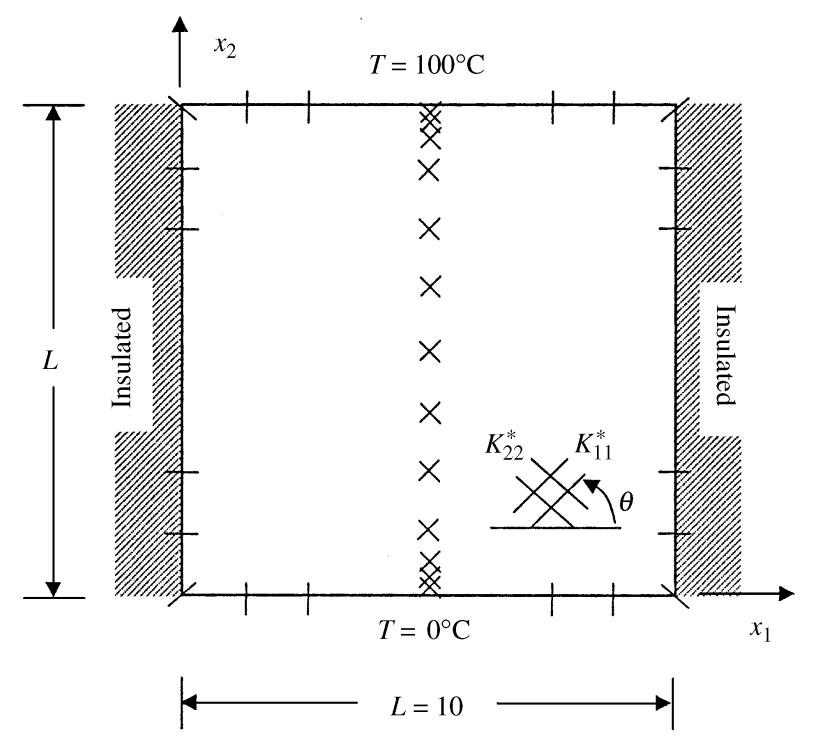

Fig. 1 Mesh discretisation and the interior points of a square plate

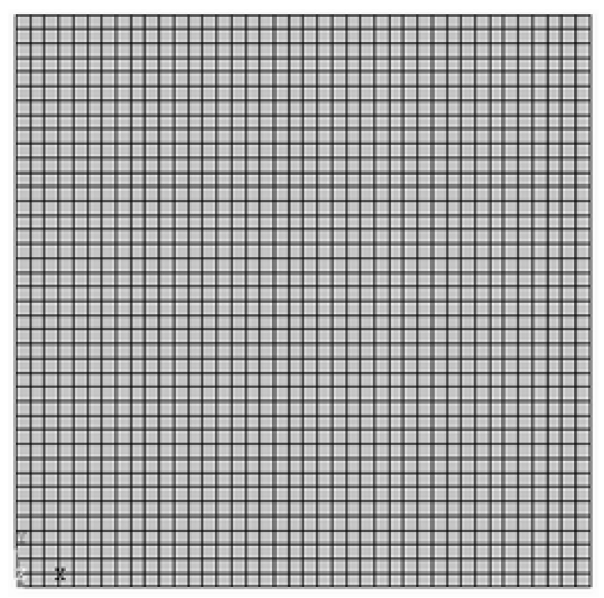

Fig. 2 FEM discretisation for the interior calculations by ANSYS

bottom surface are prescribed with temperature change $100^{\circ} \mathrm{C}$ and $0^{\circ} \mathrm{C}$, respectively, while the other two opposite sides are thermally insulated. As shown in Fig. 1, the temperature and its gradients in both directions $x_{1}, x_{2}$ are calculated using the derived BIEs for the interior points of interest, marked by cross signs. For verifying the BEM results, the problem is also investigated by ANSYS, commercial software based upon the finite element method. Also shown in Fig. 1 is the BEM mesh discretisation used for the present analysis, where a total of 20 quadratic elements are used. For the FEM analysis in ANSYS, the FEM discretisation needs relatively fine meshes in order to get data at corresponding points near the boundary. Although an adaptive discretisation with fewer meshes may be developed to meet such a requirement, a less straightforward scheme shall be

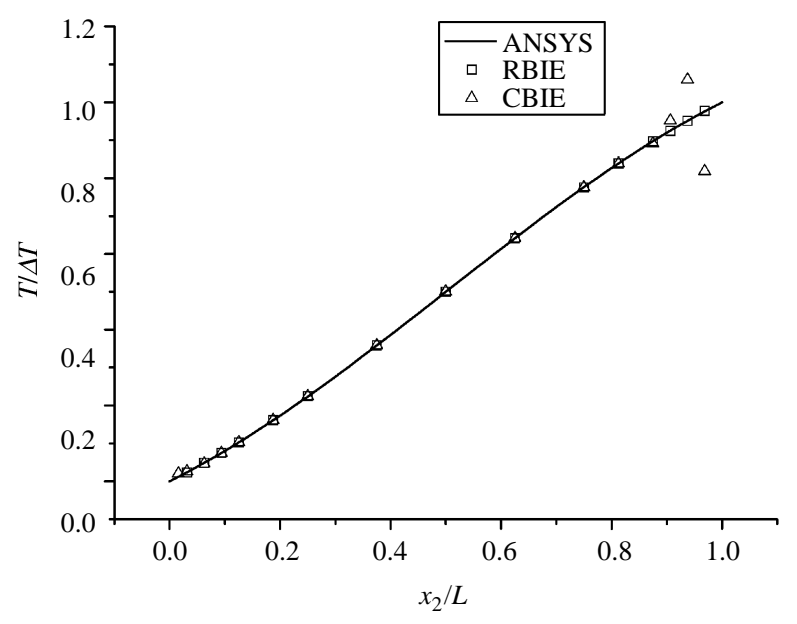

Fig. 3 Normalized temperature, $T / \Delta T$, at the interior points of interest

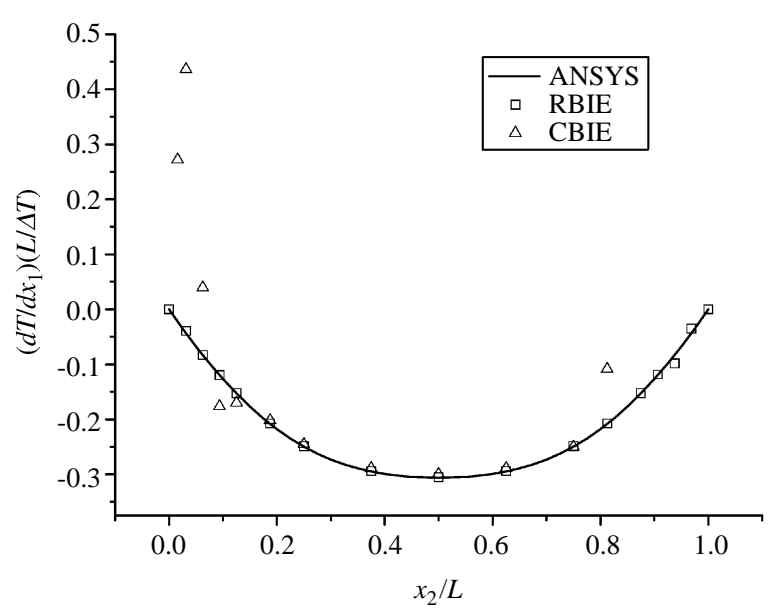

Fig. 4 Normalized temperature gradient, $d T / d x_{1} \cdot L / \Delta T$, at the interior points of interest

involved to reduce the mesh loading. Simply for the purpose of verification, a simple discretisation with constant intervals is applied. Fig. 2 shows the FEM domain discretisation used in the current analysis by ANSYS, where a total amount of 1600 PLANE42 elements are applied. For comparisons, the computations of the thermal field are carried using the present regularized BIE (RBIE) and also the conventional BIE (CBIE). The computed $T, d T / d x_{1}$, and $d T / d x_{2}$ are normalized and plotted in Figs. 3, 4, and 5, respectively. From Fig. 3, it is seen that the errors caused by the CBIE for those points near the boundary are not so obvious. This is because the singularity orders of the temperature BIE are not so strong as those in the BIE for temperature gradients. In Figs. 4 and 5, the data of CBIE for points near the top surface are not shown since they are off-scale with large errors. As seen from these figures, the results of RBIE agree very well 


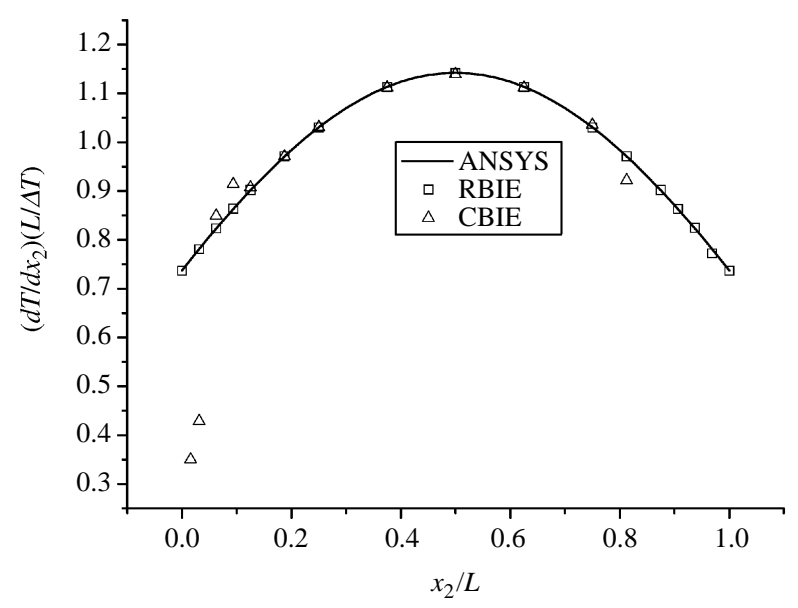

Fig. 5 Normalized temperature gradient, $d T / d x_{2} \cdot L / \Delta T$, at the interior points of interest

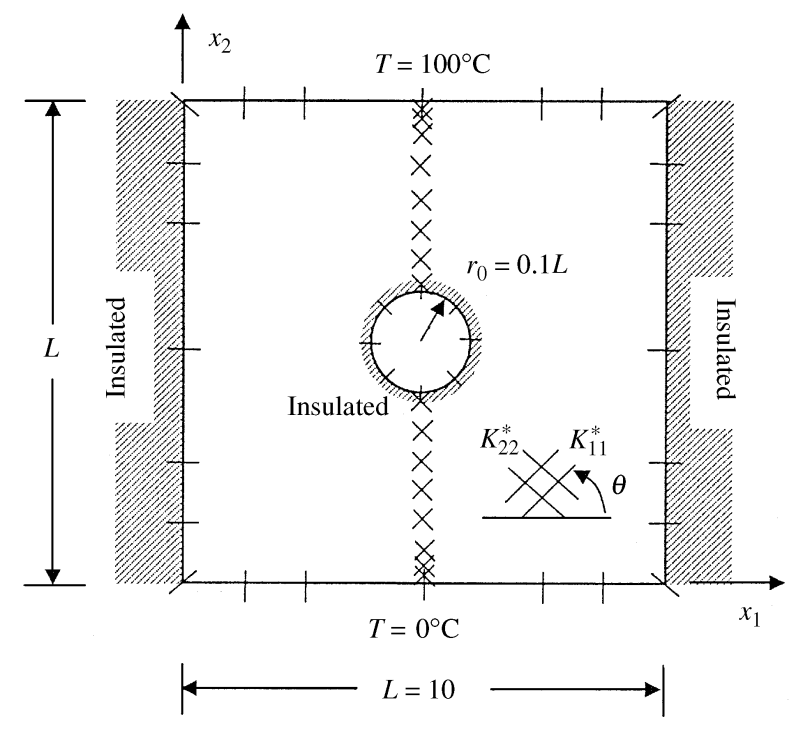

Fig. 6 The BEM meshes used for a doubly connected region and the interior points

with the analyses by ANSYS, while the CBIE fails to give satisfactory values at points near the boundary. From the results, it was observed that different sensitivities of the boundary fluxes in the normal and tangent directions strongly depend on the orientation of the principal axes. The sensitivities for isotropic media were observed by Igarashi and Honma (1993).

Another problem considered is a doubly connected glass/epoxy plate as shown in Fig. 6. As prescribed for the previous problem, all outside surfaces have the same boundary conditions. Additionally, the inside hole is thermally insulated. The principal axes are assumed to have a rotation angle $\theta=45^{\circ}$. For the BEM modeling, the domain is discretised into 32 quadratic isoparametric elements using the Lagrange interpolation. The same problem is also analyzed using

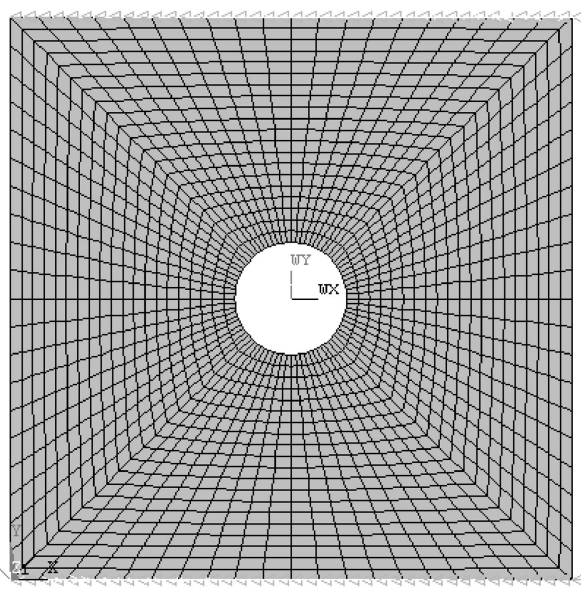

Fig. 7 The domain discretisation used for the FEM analysis for the second example

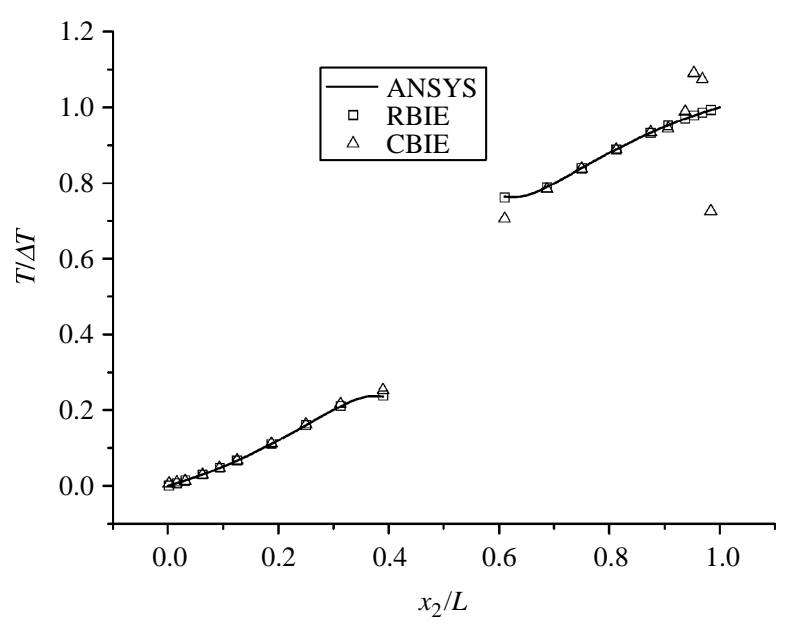

Fig. 8 Normalized temperature at the interior points for the second example

ANSYS, where a total of 1600 PLANE42 elements are applied as shown in Fig. 7. For the interior calculations at points near the boundary, relatively refined meshes are used for the FEM discretisation. Fig. 8 shows the distribution of the normalized temperature at the interior points. The normalized temperature gradients, $d T / d x_{1} \cdot L / \Delta T$ and $d T / d x_{2} \cdot L / \Delta T$, calculated using the RBIE, CBIE, and ANSYS for these interior points are plotted in Fig. 9 and Fig. 10, respectively. By observing these figures, it is apparent that the RBIE yields values in excellent agreement with those obtained by ANSYS, while CBIE fails to give satisfactory results.

\section{CONCLUDING REMARKS}

The present work deals with the interior BEM calculation of the anisotropic thermal fields at points 


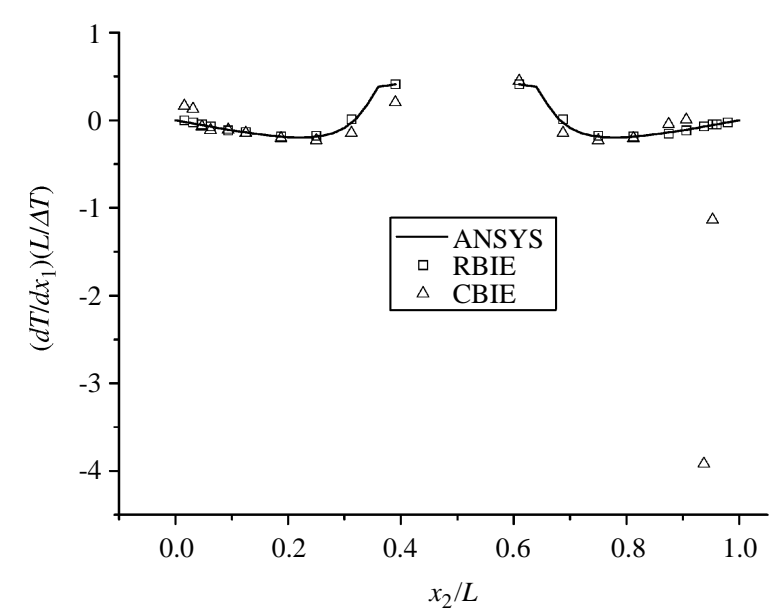

Fig. 9 Normalized temperature gradient, $d T / d x_{1} \cdot L / \Delta T$, at the interior points for the second example

near the boundary. For calculation of the interior thermal field, the boundary integrals become strongly singular for the temperature kernel and hyper-singular for the temperature gradients. The singularities are removed by the scheme of integration by parts for the integrals of the temperature kernel and by a semianalytical approach for those associated with temperature gradients. The integral equations, derived for generally high order elements, are tested and work perfectly no matter how closely the source point approaches the integration element. The successful implementation of the proposed scheme is illustrated by numerical examples, where results are verified by ANSYS analyses.

\section{ACKNOWLEDGMENTS}

The authors gratefully acknowledge the financial support of the National Science Council of Taiwan (Grant No.: NSC94-2212-E-035-011).

\section{REFERENCES}

Chang, Y. P., 1977, “Analytical Solution for Heat Conduction in Anisotropic Media in Infinite, Semi-Infinite, and Two-Placebounded Regions," International Journal of Heat and Mass Transfer, Vol. 20, pp. 1019-1028.

Chen, H. B., Lu, P., and Schnack, E., 2001, "Regularized Algorithms for The Calculation of Values on and Near Boundaries in 2D elastic BEM," Engineering Analysis with Boundary Elements, Vol. 25, No. 10, pp. 851-876.

Chen, J. T., and Hong, H. K., 1994, "Dual Boundary Integral Equations at a Corner Using Contour Approach Around Singularity," Advances in Engineering Software, Vol. 21, No. 3, pp. 169-178.

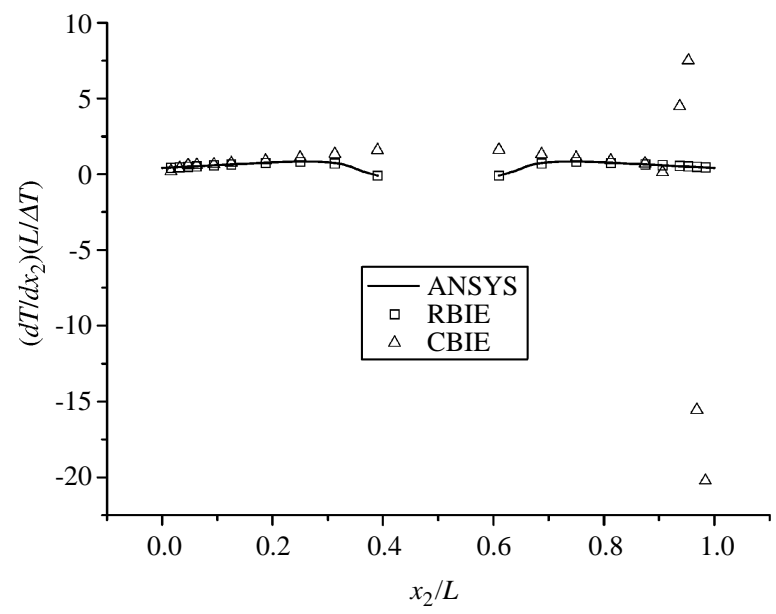

Fig. 10 Normalized temperature gradient, $d T / d x_{2} \cdot L / \Delta T$, at the interior points for the second example

Chen, J. T., and Hong, H. K., 1999, "Review of Dual Boundary Element Methods with Emphasis on Hypersingular Integrals and Divergent Series," Applied Mechanics Reviews, ASME, Vol. 52, No. 1, pp. 17-33.

Chen, J. T., Shen, W. C., and Wu, A. C., 2006b, "Nullfield Integral Ratios for Stress Field Around Circular Holes Under Anti-plane Shear," Engineering Analysis with Boundary Elements, Vol. 39, No. 3, pp. 205-217.

Chen, J. T., Hsiao, C. C., and Leu, S. Y., 2006a, "Nullfield Equation Approach for Plate Problems with Circular Holes," to appear in ASME, Journal of Applied Mechanics, Vol. 73.

Cruse, T. A., and Aithal, R., 1993, "Non-singular Boundary Integral Equation Implementation," International Journal of Numerical Method In Engineering, Vol. 36, pp. 237-254.

Huang, Q., and Cruse, T. A., 1993, "Some Notes on Singular Integral Techniques in Boundary Element Analysis," International Journal of Numerical Method In Engineering, Vol. 36, pp. 2643-2659.

Igarashi, H., and Honma, T., 1993, "A Boundary Element Analysis of Magnetic Fields Near Surface," Boundary Element Technology VIII, Comp. Mech. Publication, United Kingdom.

Kisu, H., and Kawahara T., 1988, "Boundary Element Analysis System Based on a Formulation with Relative Quantity," Boundary Elements X, edited by C.A. Brebbia, Vol. 1, pp. 111-121, Springer-Verlag, United Kingdom.

Krishnasamy, G., Rizzo, F. J., and Liu, Y. J., 1994, "Boundary Integral Equations for Thin Bodies," International Journal of Numerical Method In Engineering, Vol. 37, pp. 107-121.

Liu, Y. J., Zhang, D., and Rizzo, F. J., 1993, "Nearly Singular and Hypersingular Integrals in the 
Boundary Element Method," Boundary Elements $X V$, C.A. Brebbia and J.J. Rencis eds., Computational Mechanics Publication, Southampton, NY, USA, pp. 453-468.

Liu, Y. J., 1998, “Analysis of Shell-like Structures by the Boundary Element Method Based on 3-D Elasticity: Formulation and Verification," International Journal of Numerical Method In Engineering, Vol. 41, No. 3, pp. 541-558, United Kingdom.

Lutz, E. D., Ingraffea, A. R., and Gray, L. J., 1992, “Use of Simple Solutions' for Boundary Integral Methods in Elasticity and Fracture Analysis," International Journal of Numerical Method In Engineering, Vol. 35, pp. 1737-1751.

Mulholland, G. P., and Gupta, B. P., 1977," Heat Transfer in a Three-dimensional Anisotropic Solid of Arbitrary Shape," Journal of Heat Transfer, Vol. 99, pp. 135-137.

Nakagawa, N., 1993, "Near-surface Field Evaluation in Two-phase Helmholtz Problem," IABEM-93 Symposium, Braunschweig, Germany.

Sladek, V., Sladek J., and Tanaka, M., 2001, "Numerical Integration of Logarithmic and Nearly Logarithmic Singularity in BEMs," Applied Mathematical Modeling, Vol. 25, pp. 901-922.

Shiah, Y. C., and Tan, Y. C., 1998, "BEM Treatment of Two-dimensional Anisotropic Field Problems by Direct Domain Mapping," Engineering Analysis with Boundary Elements, Vol. 20, pp. 347-351. Shiah Y. C., and Shih, Y. S., 2005, "Heat Conduction in An Anisotropic Media With Thermal Barrier Coating", accepted to be published in the International communications in Heat and Mass Transfer, Elsevier Science Ltd., Manuscript No. ICHMT 1480.

Tanaka, V., Sladek, V., and Sladek, J., 1994, "Regularization Techniques Applied to BEM," Applied Mechanics Review, ASME, Vol. 47, No. 10, pp. 457-499.

Tauchert, T. R., and Akoz, A. Y., 1975, "Stationary Temperature and Stress Fields in An Anisotropic Elastic Slab," Journal of Applied Mechanics, Vol. 42, pp. 647-650.

Zhou, H., Niu, Z., and Wang, X., 2003, "The Regularization of Nearly Singular Integrals in the BEM of Potential Problems," Applied Mathematics and Mechanics, Vol. 24, No. 10, pp. 1208-1214.

Manuscript Received: Nov. 28, 2005

Revision Received: Jun. 07, 2006

and Accepted: Jun. 21, 2006 